\title{
Parálisis periódica tirotóxica: revisión de la literatura y reporte de caso en un hospital universitario mexicano privado de alta especialidad
}

\author{
Manuel A. Sierra ${ }^{1,2}$, Wallace R. Muñoz-Castañeda ${ }^{1 *}$, Carlos Tolsa $^{1}$, Aldo E. Lara ${ }^{1}$, Raúl Medina ${ }^{1}$ y \\ Carolina Vega ${ }^{2}$ \\ ${ }^{1}$ Departamento de Neurología; ${ }^{2}$ Departamento de Medicina Interna, Hospital Clínica Médica Sur; ${ }^{3}$ Departamento de Neurología, Instituto Nacional \\ de Neurología y Neurocirugía, Hospital Manuel Velasco Suárez. Ciudad de México, México
}

\begin{abstract}
Resumen
La parálisis periódica tirotóxica (PTT) es una complicación mortal de la tirotoxicosis caracterizada por hipopotasemia súbita, parálisis muscular y tirotoxicosis. La PTT, aunque cada vez es más frecuente en nuestro medio, todavía no diagnostica de forma oportuna debido a la falta de familiaridad y la sutileza de la sintomatología referida. El reconocimiento temprano por parte del médico de primer contacto puede prevenir la mortalidad de dicha enfermedad. Se presenta un caso típico de parálisis tirotóxica en un hospital universitario privado mexicano y se realiza una revisión de la literatura más actual. Realizamos una revisión de la literatura en PubMed y EMBASE. Se analizaron características clínicas, bioquímicas y diagnósticas, la terapéutica y el pronóstico. La PPT es una entidad clínica que se presenta de forma rara en la población mexicana, sin embargo es importante encontrarse familiarizado con el diagnóstico ya que el tratamiento es diferente al de otras parálisis periódicas hipopotasémicas.
\end{abstract}

Palabras clave: Parálisis. Tirotoxicosis. Periódica. Hipopotasemia. Arreflexia.

\section{Thyrotoxic periodic paralysis: review of the literature and case report in a high specialty Mexican university hospital}

\begin{abstract}
Thyrotoxic periodic paralysis (TPP) is a fatal complication of thyrotoxicosis, characterized by sudden hypokalemia, muscle paralysis and thyrotoxicosis. TPP, although it is becoming more frequent in our country, is still not diagnosed in a timely manner due to the lack of familiarity and the subtlety of the referred symptomatology. Early recognition by the first contact physician can prevent the mortality of said disease. A typical case of thyrotoxic paralysis is presented in a private Mexican university hospital and a review of the most current literature is made. We conducted a review of the literature in PubMed and EMBASE. Clinical, biochemical, diagnostic, therapeutic and prognostic characteristics were analyzed. TPP is a clinical
\end{abstract}

\section{Correspondencia:}

*Wallace Rafael Arturo Muñoz-Castañeda

Departamento de Medicina Interna

Hospital Médica Sur

Sillón de Mendoza, 110

Col. Toriello Guerra, Del. Tlalpan $\quad$ Fecha de recepción: 04-02-2018

Ciudad de México, México

Fecha de recepción: 04-02-2018

Disponible en internet: 18-06-2019

E-mail: dr.wallace4719@gmail.com

DOI: 10.24875/RMN.M19000046

Rev Mex Neuroci. 2019;20(3):149-154

www.revmexneurociencia.com

1665-5044/@ 2018. Academia Mexicana de Neurología A.C. Publicado por Permanyer México. Este es un artículo Open Access bajo la licencia CC BY-NC-ND (http://creativecommons.org/licenses/by-nc-nd/4.0/). 
Rev Mex Neuroci. 2019;20

entity that occurs rarely in the Mexican population, however it is important to be familiar with the diagnosis since the treatment is different from other periodic hypokalemic paralysis..

Key words: Paralysis. Thyrotoxicosis. Periodic. Hypokalemia. Areflexia.

\section{Introducción}

La asociación de parálisis periódica y tirotoxicosis fue documentada por Rosenfeld en 1902. Mundialmente, los asiáticos orientales son la población más afectada; en ellos la incidencia es de alrededor del $2 \%$. En México se desconoce la incidencia real ${ }^{1,2,3}$. La PPT se caracteriza por la tríada de: hipopotasemia grave, debilidad muscular profunda y presencia de tirotoxico$\mathrm{sis}^{3,4}$. La hipopotasemia en la PPT resulta de un desplazamiento intracelular de potasio inducido por la sensibilización de la bomba Na+/K+-ATPasa por la hormona tiroidea ${ }^{4}$. El diagnóstico precoz no solo ayuda en el tratamiento definitivo con betabloqueadores no selectivos y en la corrección del hipertiroidismo, sino que también previene el riesgo de hiperpotasemia de rebote debido al reemplazo excesivo de potasio. La TTP es curable una vez que se logra un estado eutiroideo ${ }^{4}$.

\section{Presentación de caso clínico}

Mujer de 33 años de edad, hispana, sin antecedentes heredofamiliares de importancia. Niega antecedentes personales patológicos, incluyendo el consumo de medicamentos de uso crónico.

Inició su padecimiento actual en noviembre del 2017 a las 20:00 h, al presentar de forma súbita parestesias en extremidades inferiores en región distal, bilateral y simétrica con irradiación a muslos. Tres horas después se agregó disminución de la fuerza en ambas extremidades inferiores que impedía la deambulación, por lo cual acude a otra institución (no especificada) donde se administró ketorolaco $30 \mathrm{mg}$ vía intravenosa dosis única sin mejoría de la sintomatología. Seis horas después del inicio del padecimiento se agrega pérdida de fuerza de extremidades superiores, así como cervicalgia, bradilalia y disnea de pequeños esfuerzos. Por lo anterior sus familiares la traen a Urgencias de nuestra institución.

A su llegada a Urgencias se encontró estable hemodinámicamente, signos vitales dentro de parámetros normales. Alerta, orientada, facies álgica, pares craneales sin alteraciones, cuadriplejia, arreflexia, sin presencia de reflejos patológicos. Cardiopulmonar sin alteraciones. Abdomen asignológico. Al interrogatorio directo refirió episodios aislados de diaforesis profunda persistente de $48 \mathrm{~h}$ de evolución, pérdida de $15 \mathrm{~kg}$ de peso no intencionada de 3 meses de evolución; cefalea y palpitaciones, así como diarrea sin moco ni sangre de 1 semana de evolución.

En los laboratorios generales destacó: hiperglucemia (195.4 mg/dl) e hipopotasemia severa (1.42). El electrocardiograma evidenció bloqueo AV de segundo grado Mobitz II. Se decidió colocación de catéter venoso central, así como reposición vía intravenosa de potasio. Punción lumbar sin alteraciones (glucosa 86, proteínas 23 , sin microorganismos teñidos con Gram). Por los hallazgos anteriores se decidió traslado a la Unidad de Terapia Intensiva para continuar abordaje y tratamiento.

Después de la reposición de potasio la paciente evolucionó con hiperreflexia generalizada y recuperación de la fuerza muscular (4/5) en las 4 extremidades. Como principal sospecha de una parálisis periódica de causa tirotóxica se solicitó perfil tiroideo, así como anticuerpos antitiroideos, reportando TSH suprimida y hormonas tiroideas aumentadas ( TSH $<0.003$, T32.85, T4L 4.27, T4 16.71, anticuerpos antiperoxidasa positivos). Se calculó el gradiente transtubular de potasio, siendo del $12 \%$ (sugerente de pérdida renal) y también la puntuación en la escala de Burch-Wartofsky, siendo de 35 (elevado riesgo para tormenta tiroidea incipiente). Se inició tratamiento con tiamazol, propranolol y colestiramina, con buena tolerancia. Posteriormente presentó cifra de potasio de control dentro de rangos normales (5.16), fuerza muscular en las cuatro extremidades de manera total. Con lo anterior se integró hipertiroidismo autoinmunitario con parálisis hipopotasémica. La paciente fue egresada $48 \mathrm{~h}$ después de su ingreso con adecuada evolución, asintomática y con laboratorios dentro de rangos normales.

\section{Definición}

La parálisis tirotóxica (PT) es una complicación potencialmente fatal del hipertiroidismo (tirotoxicosis), reversible y caracterizada por parálisis muscular e hipopotasemia severa.

La parálisis periódica tirotóxica (PPT) corresponde a un tipo de parálisis hipopotasémica que constituye la principal causa de parálisis periódica hipopotasémica 
en países asiáticos, a diferencia de la parálisis periódica hipopotasémica familiar, que es la causa más común en países occidentales de herencia autosómica dominante $e^{1,2,3}$.

\section{Epidemiología}

Debido a su baja prevalencia en los países occidentales y a la sutileza de su presentación, la tirotoxicosis a menudo no se reconoce ${ }^{3}$. A pesar de que se ve comúnmente en la enfermedad de Graves, la PPT no se encuentra relacionada con la etiología, la gravedad y la duración de la tirotoxicosis. Generalmente no hay antecedentes familiares de parálisis periódica. Algunos subtipos de antígenos HLA tales como DRw8, A2, BW22, Aw19, B17 B5 y Bw46 en ciertas poblaciones étnicas pueden hacerlos más susceptibles al PPT ${ }^{4,5}$.

La incidencia de PPT en pacientes tirotóxicos chinos $y$ japoneses se ha informado en el 1.8 y el $1.9 \%$, respectivamente, y en EE.UU. entre el 0.1 y el $0.2 \%$. En México no existen datos sobre la incidencia de esta relación. La relación entre hombres y mujeres va de 17:1 a 70:1, a pesar de que el hipertiroidismo es más común en las mujeres (proporción de mujeres a hombres de 9:1) $)^{4,5,6}$.

\section{Etiología}

La causa más común de tirotoxicosis es la enfermedad de Graves, sin embargo, se han reportado casos de PT en pacientes con bocio tóxico nodular, tirotoxicosis inducida por yodo, tiroiditis linfocítica, adenomas secretores de tirotropina e incluso tiroiditis inducida por amiodarona ${ }^{1}$.

\section{Fisiopatogenia}

La fisiopatología de la PPT es multifactorial y aún no se comprende por completo. Se sabe que la PPT se debe a la disminución de potasio intracelular y no a la depleción de potasio corporal. Muchas teorías han tratado de definir la fisiopatología de la PPT. Una de las teorías más aceptadas es el incremento de la actividad de la bomba $\mathrm{Na}+/ \mathrm{K}+-\mathrm{ATPasa}$, encontrándose una tasa de actividad de hasta un $80 \%$ mayor en los pacientes diagnosticados con PPT. La bomba Na+/K+-ATPasa se activa mediante distintos estímulos entre los cuales destacan: la insulina, el ejercicio, la estimulación adrenérgica, las hormonas tiroideas y la testosterona. Los estrógenos y la progesterona han demostrado disminuir la actividad de la bomba $\mathrm{Na+/K+-ATPasa,} \mathrm{lo} \mathrm{que}$

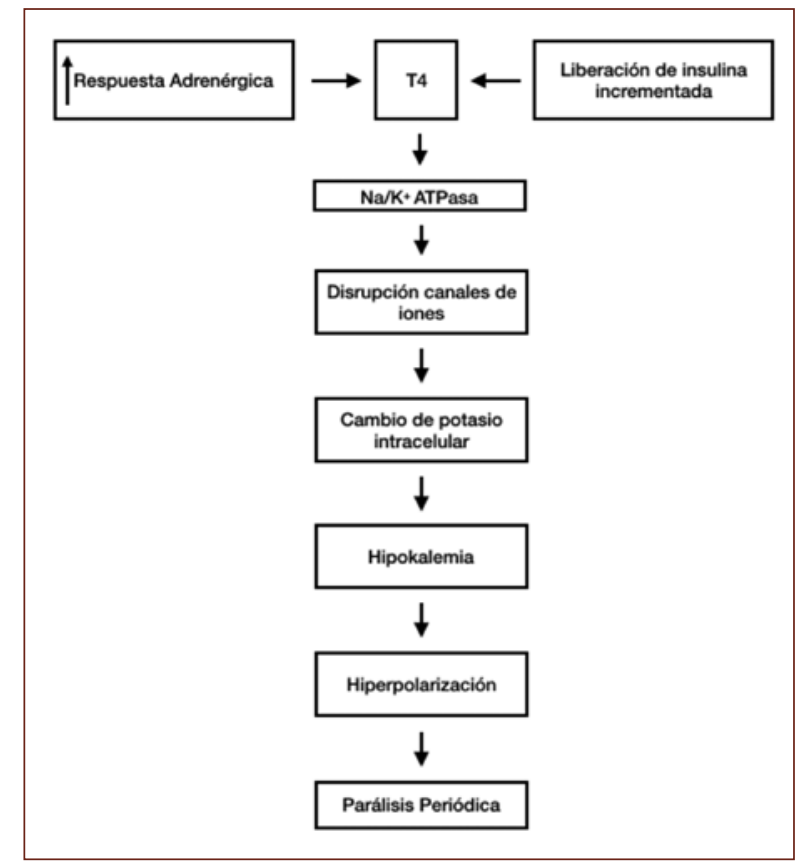

Figura 1. Mecanismo de la parálisis periódica tirotóxica (adaptada de Abhishek, et al., 2004²).

podría explicar la mayor incidencia de PPT en el sexo masculino ${ }^{7,8}$ (Fig. 1). Actualmente existen mutaciones bien conocidas que influyen en la fisiopatogenia de la PPT; un ejemplo claro es la mutación de Kir 2.6, un canal de potasio regulado por hormonas tiroideas localizado en el músculo esquelético. El hipertiroidismo puede provocar un estado hiperadrenérgico que puede conducir a la activación de la bomba de $\mathrm{Na}+/ \mathrm{K}+-\mathrm{ATPa}$ sa y dar como resultado la captación celular de potasio. Se ha encontrado que los pacientes con PPT tienen hiperinsulinemia durante los episodios de parálisis. Esto puede explicar los ataques después de las comidas con alto contenido de carbohidratos ${ }^{12}$.

\section{Manifestaciones clínicas y diagnóstico}

La parálisis periódica puede manifestarse con cualquier condición asociada con hipopotasemia (Tabla 1). El diagnóstico de una PPT se basa en la presentación de parálisis flácida de comienzo súbito en el contexto de hipopotasemia e hipertiroidismo. Este cuadro clínico suele presentarse en individuos de la tercera a la quinta década de la vida, con una mayor incidencia en hombres, lo que contrasta con la parálisis periódica familiar (PPF), que suele tener un inicio más temprano ${ }^{9}$.

La parálisis se caracteriza por debilidad muscular de inicio abrupto de predominio proximal, principalmente en miembros inferiores. En la mayoría de los casos es simétrica y está acompañada de hiporreflexia/arreflexia. 
Tabla 1. Diagnósticos diferenciales de parálisis hipopotasémica

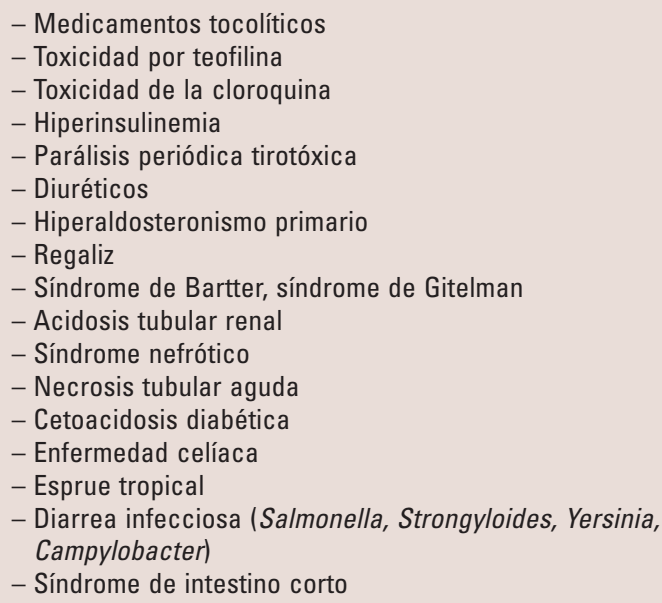

Dicha parálisis puede llegar a progresar hasta cuadriparesia. De manera excepcional se ven afectados la sensibilidad, las funciones mentales y los músculos oculares, bulbares o respiratorios ${ }^{10}$. Dichos episodios pueden tener una duración de minutos a días sin tratamiento. En algunos casos se ha llegado a reportar que horas a días previos a los episodios los pacientes pueden manifestar mialgias, rigidez, calambres o debilidad leve que se autolimitan antes de presentar la parálisis ${ }^{11}$.

La mayoría de los casos reportados de esta parálisis suelen ser en los meses calurosos del año y su horario de presentación es predominantemente de la noche a primeras horas de la mañana ${ }^{12}$. Una de las características clínicas que podría ayudar a diferenciar la PPF y la PPT es la presencia de taquicardia y la tendencia a la hipertensión sistólica en los pacientes con diagnóstico de PPT ${ }^{13}$

La hipopotasemia suele estar presente en la gran mayoría de los episodios de parálisis y el nivel elevado de potasio sérico $(5 \mathrm{mmol} / \mathrm{l})$ suele correlacionar con la gravedad del cuadro. En la mayoría de las series de casos el nivel de potasio sérico promedio llega a rondar entre 1.9 y $2.4 \mathrm{mmol} /$; sin embargo, se han reportado casos con potasio sérico dentro de valores normales o con niveles de hipopotasemia extremadamente bajos. La excreción urinaria de potasio suele estar disminuida, lo que ayuda a diferenciar la PPT de otras causas de parálisis hipopotasémica. Esto debe ser demostrado utilizando la relación potasio/creatinina y el gradiente transtubular de potasio, ya que la cuantificación aleatoria de potasio urinario suele ser un método inexacto para calcular la excreción renal de potasio ${ }^{13,14,15}$.
Otras alteraciones de electrólitos séricos descritas en estos pacientes suelen ser la hipofosfatemia e hipomagnesemia leves ${ }^{15}$. A nivel urinario se ha llegado a reportar que los pacientes con PPT presentan aumento del índice calcio/creatinina y disminución del índice fosfato/creatinina. Basándose en estos hallazgos, Lin, et al. proponen que la relación calcio urinario/ fosfato urinario con un valor mayor de $1.7 \mathrm{mg} / \mathrm{mg}$ podría tener una sensibilidad y especificidad del 100 y el 96\% respectivamente para el diagnóstico de PPT en relación con otras causas de parálisis hipopotasémica. La presencia de hipertiroidismo, caracterizado por un perfil tiroideo sérico con T3 y/o T4 aumentadas con TSH disminuida, es un criterio fundamental para el diagnóstico de esta entidad. Sin embargo, no se ha encontrado relación entre los niveles de hormonas tiroideas y la gravedad de la parálisis ${ }^{10,11}$.

En diferentes estudios se ha aproximado que más del $50 \%$ de los pacientes presentan PPT sin contar con un diagnóstico previo o cuadro clínico sugerente de hipertiroidismo. Dicho hallazgo fue corroborado en un estudio en el que utilizando el índice de Wayne se determinó que solo el $17 \%$ de los pacientes de dicha población presentaba síntomas o signos francos de hipertiroidismo (índice de Wayne $>19$ ). Mientras que el $38 \%$ de los pacientes se podían clasificar como clínicamente eutiroideos (índice de Wayne $<11$ ) ${ }^{12}$.

Dadas las alteraciones electrolíticas características de esta entidad, los cambios electrocardiográficos son comunes en esta enfermedad. Conocer la prevalencia de dichos cambios es importante para el clínico, para el correcto tratamiento y vigilancia del paciente. Dichos cambios varían en frecuencia y van desde la taquicardia sinusal hasta arritmias fatales y usualmente no correlacionan con la intensidad de los síntomas ${ }^{15}$.

En la mayoría de los casos, se observan cambios consistentes con niveles séricos bajos de potasio como depresión del segmento ST, taquicardia sinusal y ondas U. En algunas series se reportan arritmias severas, las cuales resultan menos frecuentes pero suelen tener desenlaces fatales como bloqueo AV de segundo grado, fibrilación ventricular y taquicardia ventricular ${ }^{16}$. Por lo anterior, se recomienda monitoreo continuo electrocardiográfico de estos pacientes en una unidad crítica, así como controles de electrólitos séricos de manera seriada $^{17}$. En la mayoría de los pacientes los estudios electrodiagnósticos como la electromiografía (EMG), no son necesarios. Sin embargo, la EMG realizada durante los episodios de debilidad típicamente revelan cambios miopáticos con amplitud disminuida del potencial de acción muscular (CMAP), así como disminución 
del reclutamiento motor o silencio eléctrico dependiendo de la severidad de la debilidad. Otros hallazgos descritos menos frecuentes incluyen aumento de la actividad de inserción, aumento de los potenciales polifásicos de la unidad motora y reducción de la velocidad de conducción de la fibra muscular. Dichos cambios no se modifican con la estimulación con epinefrina. En cuanto al estudio de la conducción nerviosa, de manera general resultan sin alteraciones.

En algunos casos, la actividad física puede desencadenar ataques de debilidad, con cambios consistentes en la EMG. Una técnica de EMG que puede usarse para ayudar a confirmar el diagnóstico entre ataques es la «prueba de ejercicio». Los CMAP que siguen un solo estímulo eléctrico supramáximo se registran en reposo y posteriormente de manera seriada con el ejercicio. Una reducción gradual de más del $40 \%$ en la amplitud motora evocada después de 30 a 40 min es compatible con un diagnóstico de parálisis periódica, pero no es específico. Se ha reportado una sensibilidad del $71 \%$ de esta prueba, pero disminuye cuando los ataques no han sido recientes. Todos estos cambios mejoran cuando se restablece la función tiroidea ${ }^{18-24}$.

\section{Tratamiento}

El tratamiento inicial en el paciente con parálisis periódica secundaria a tirotoxicosis se centra en mejorar los niveles séricos de potasio. Es importante destacar que los pacientes con PPT no tienen un déficit del potasio corporal total, por lo que la administración de potasio debe ser controlada para evitar hiperpotasemia de rebote ${ }^{25}$.

La suplementación con potasio para el tratamiento de la PPT ha demostrado disminuir el tiempo de recuperación y disminuir complicaciones cardiopulmonares. La administración de potasio puede llevarse a cabo por vía oral o vía intravenosa. Sin embargo, existe una serie de casos en los cuales se observó un menor tiempo de respuesta en los sujetos que fueron tratados con potasio intravenoso en comparación con potasio vía oral. Se recomienda que la velocidad de infusión de cloruro de potasio no sea mayor de $10 \mathrm{mmol} / \mathrm{h}$ y se ha llegado a recomendar no superar una dosis de 50 mmol en $24 \mathrm{~h}$. Estas recomendaciones se basan en publicaciones que llegan a reportar hiperpotasemia de rebote en el 40 hasta el $80 \%$ de los pacientes que reciben más de $90 \mathrm{mmol}$ de cloruro de potasio en un periodo de $24 \mathrm{~h}^{26-30}$.

Aunque no existen ensayos clínicos controlados que demuestren el beneficio del uso de betabloqueadores no selectivos en el tratamiento agudo de la PPT, existen múltiples reportes de casos en los cuales la administración de propranolol, ya sea por vía intravenosa o vía oral, se ha asociado a reversibilidad del cuadro, tanto como terapia inicial como en pacientes en los que no se observó mejoría posterior a la reposición de potasio. Dicho tratamiento puede ser iniciado de manera simultánea o utilizarse en caso de resistencia al tratamiento inicial. Las dosis mencionadas en diferentes reportes van de $3 \mathrm{mg} / \mathrm{kg}$ de propranolol vía oral y de $1 \mathrm{mg}$ de propranolol intravenoso cada $10 \mathrm{~min}$ sin superar la dosis total de $3 \mathrm{mg}$. En la mayoría de los casos no se ha asociado el uso de betabloqueadores no selectivos a hiperpotasemia de rebote ${ }^{31,32}$.

\section{Tratamiento preventivo}

El objetivo primordial del tratamiento preventivo se centra en lograr el retorno al eutiroidismo, dicha medida evitará la recurrencia de los episodios de parálisis. La tasa de recurrencia puede llegar a ser de hasta el $60 \%$ en los pacientes que aún no se encuentran eutiroideos, presentando el mayor riesgo los primeros 3 meses tras el diagnóstico de PPT. El tratamiento del hipertiroidismo se puede llevar a cabo por cualquiera de los métodos estándar de tratamiento: farmacológico, quirúrgico o yodo radioactivo. Existen estudios que han demostrado que según la etiología del hipertiroidismo pueden existir terapéuticas más apropiadas que otras en cuestión de disminuir la recurrencia de la parálisis. Siendo los más recomendados los métodos definitivos como la ablación con yodo radioactivo o la tiroidectomía. Sin embargo, existen asociaciones de recurrencia de la parálisis posterior al uso de yodo radioactivo ${ }^{33,34}$.

En el periodo en el que se consigue la normalización de las hormonas tiroideas, el uso de betabloqueadores no selectivos puede disminuir la frecuencia de los episodios de parálisis. Aunque no existe una dosis establecida de propranolol, en un estudio clínico la dosis de $40 \mathrm{mg}$ de propranolol vía oral cada $6 \mathrm{~h}$ se asoció a una disminución del $71 \%$ de la PPT inducida por carbohidratos. De igual forma se tiene que evitar la exposición a condiciones predisponentes: dieta alta en carbohidratos, consumo excesivo de sal, alcohol o ejercicio intenso mientras los niveles séricos de hormonas tiroideas no se encuentren dentro de los valores normales. Por último, es importante mencionar que el uso de acetazolamida y la suplementación de potasio vía oral como tratamiento preventivo puede precipitar los episodios de parálisis, en contraste con su utilidad en la parálisis periódica hipopotasémica familiar ${ }^{35,36}$. 


\section{Financiamiento}

La presente investigación no ha recibido ayudas específicas provenientes de agencias del sector público, sector comercial o entidades sin ánimo de lucro.

\section{Conflicto de intereses}

Los autores declaran no tener conflicto de intereses.

\section{Bibliografía}

1. Morales-Victorino N, Guevara-Arnal L, Almeda-Valdés P. Parálisis tirotóxica: informe de un caso y revisión de la literatura. Rev Medica Sur. 2008;15(2):81-5.

2. Vijayakumar A, Ashwath G, Thimmappa D. Thyrotoxic periodic paralysis: Clinical J Thyroid Res. 2014;2014:649502.

3. Patel H, Wilches LV, Guerrero J. Thyrotoxic periodic paralysis: Diversity in America. J Emerg Med. 2014;46(6):760-2.

4. Lam L, Nair RJ, Tingle L. Thyrotoxic periodic paralysis. Proc (Bayl Univ Med Cent). 2006;19(2):126-9.

5. Pothiwala P, Levine SN. Analytic review: thyrotoxic periodic paralysis: a review. J Intensive Care Med. 2010;25:71.

6. Pompeo A, Nepa A, Maddestra M, Feliziani V, Genovesi N. Thyrotoxic hypokalemic periodic paralysis: An overlooked pathology in western countries. Eur J Intern Med. 2007;18:380-90.

7. Guerra M, Rodriguez Del Castillo A, Battaner E, Mas M. Androgens stimulate preoptic area $\mathrm{Na}+\mathrm{K}+\mathrm{ATP}$ ase activity in male rats. Neurosci Lett. 1987;78(1):97-100.

8. Feely J. Potassium shift in thyrotoxic periodic paralysis. Postgrad Med J. 1981;57:238-9

9. Lee KO, Taylor EA, Oh VM, Cheah JS, Aw SE. Hyperinsulinemia in thyrotoxic hypokalemic periodic paralysis. Lancet. 1991;337(8749):1063-4.

10. Lin S-H. Thyrotoxic periodic paralysis. Mayo Clin Proc. 2005;80(1):99105.

11. Salih M, van Kinschot CMJ, Peeters RP, de Herder WW, Duschek EJJ, van der Linden J, et al. Thyrotoxic periodic paralysis: an unusual presentation of hyperthyroidism. Neth J Med. 2017;75(8):315-20.

12. Chang CC, Cheng CJ, Sung CC, Chiueh TS, Lee CH, Chau T, et al. A 10-year analysis of thyrotoxic periodic paralysis in 135 patients: focus on symptomatology and precipitants. Eur J Endocrinol. 2013;169(5):529-36.

13. Goldberger ZD. Images in cardiovascular medicine. An electrocardiogram triad in thyrotoxic hypokalemic periodic paralysis. Circulation. 2007:115:e179.

14. Hsu YJ, Lin YF, Chau T, Kuo SW, Lin SH. Electrocardiographic manifestations in patients with thyrotoxic periodic paralysis. Am J Med Sci. 2003:326:128.

15. Areta-Higuera JD, Algaba-Montes M, Oviedo-García AA. Parálisis periódica hipopotasémica. A propósito de un caso. SEMERGEN. 2014;40:4.

16. Fisher J. Thyrotoxic periodic paralysis with ventricular fibrillation. Arch Intern Med. 1982;142:1362-4.
17. Loh KC, Pinheiro L, Ng KS. Thyrotoxic period paralysis complicated by near-fatal ventricular arrhythmias. Singapore Med J. 2005;46:88-9.

18. Ryzen E, Servis KL, Rude RK. Effect of intravenous epinephrine on serum magnesium and free intracellular red blood cell magnesium concentrations measured by nuclear magnetic resonance. J Am Coll Nutr. 1990;9:114-9.

19. Tengan $\mathrm{CH}$, Antunes $\mathrm{AC}$, Gabbai AA, Manzano GM. The exercise test as a monitor of disease status in hypokalaemic periodic paralysis. J Neural Neurosurg Psychiatry. 2004;75:497-9.

20. Kung AWC. Thyrotoxic periodic paralysis: A diagnostic challenge. J Clin Endocrinol Metab. 2006;91:2490-5.

21. Venance SL, Cannon SC, Fialho D, Fontaine B, Hanna MG, Ptacek LJ, et al. The primary periodic paralyses: diagnosis, pathogenesis and treatment. Brain. 2006;129:8.

22. Sharma CM, Nath K, Parekh J. Reversible electrophysiological abnormalities in hypokalemic paralysis: Case report of two cases. Ann Indian Acad Neurol. 2014;17:100

23. Tengan $\mathrm{CH}$, Antunes AC, Gabbai AA, Manzano GM. The exercise test as a monitor of disease status in hypokalaemic periodic paralysis. J Neurol Neurosurg Psychiatry. 2004;75:497.

24. Kuntzer T, Flocard F, Vial C, Kohler A, Magistris M, Labarre-Vila A, et al. Exercise test in muscle channelopathies and other muscle disorders. Muscle Nerve. 2000;23:1089.

25. Lin SH, Lin YF, Halperin ML. Hypokalaemia and paralysis. QJM. 2001;94(3):133-9.

26. Cesur M, Bayram F, Temel MA, Ozkaya M, Kocer A, Ertorer ME, et al. Thyrotoxic hypokalaemic periodic paralysis in a Turkish population: three new case reports and analysis of the case series. Clinical Endocrinology. 2008;68:143-52.

27. Manoukian MA, Foote JA, Crapo LM. Clinical and metabolic features of thyrotoxic periodic paralysis in 24 episodes. Arch Intern Med. 1999;159:601.

28. Lin S-H, Chu P, Cheng C-J, Chu S-J, Hung Y-J, Lin Y-F. Early diagnosis of thyrotoxic periodic paralysis: spot urine calcium to phosphate ratio. Crit Care Med. 2006;34(12):2984-9.

29. Hsieh MJ, Lyu RK, Chang WN, Chang KH, Chen CM, Chang HS, et al. Hypokalemic thyrotoxic periodic paralysis: clinical characteristics and predictors of recurrent paralytic attacks. Eur J Neurol. 2008;15(6):559-64.

30. Lu KC, Hsu YJ, Chiu JS, Hsu YD, Lin SH. Effects of potassium supplementation on the recovery of thyrotoxic periodic paralysis. Am J Emerg Med. 2004;22(7):544-7.

31. Lin SH, Lin YF. Propranolol rapidly reverses paralysis, hypokalemia, and hypophosphatemia in thyrotoxic periodic paralysis. Am J Kidney Dis. 2001;37(3):620.

32. Shayne P, Hart A. Thyrotoxic periodic paralysis terminated with intravenous propranolol. Ann Emerg Med. 1994;24(4):736.

33. Chang RY, Lang BH, Chan AC, Wong KP. Evaluating the efficacy of primary treatment for Graves' disease complicated by thyrotoxic periodic paralysis. Int J Endocrinol. 2014;2014:949068.

34. Akar S, Comlekci A, Birlik M, Onen F, Sari I, Gurler O, et al. Thyrotoxic periodic paralysis in a Turkish male; the recurrence of the attack after radioiodine treatment. Endocr J. 2005;52(1):149-51.

35. Vijayakumar A, Ashwath G, Thimmappa D. Thyrotoxic periodic paralysis: clinical challenges. J Thyroid Res. 2014;2014:649502.

36. Yeung RT, Tse TF. Thyrotoxic periodic paralysis. Effect of propranolol. Am J Med. 1974;57(4):584-90. 